UCRL-ID-133293

\title{
High Intensity Physics with a Table-Top 20 TW Laser System
}

T. Ditmire and M. D. Perry

February 24, 1999

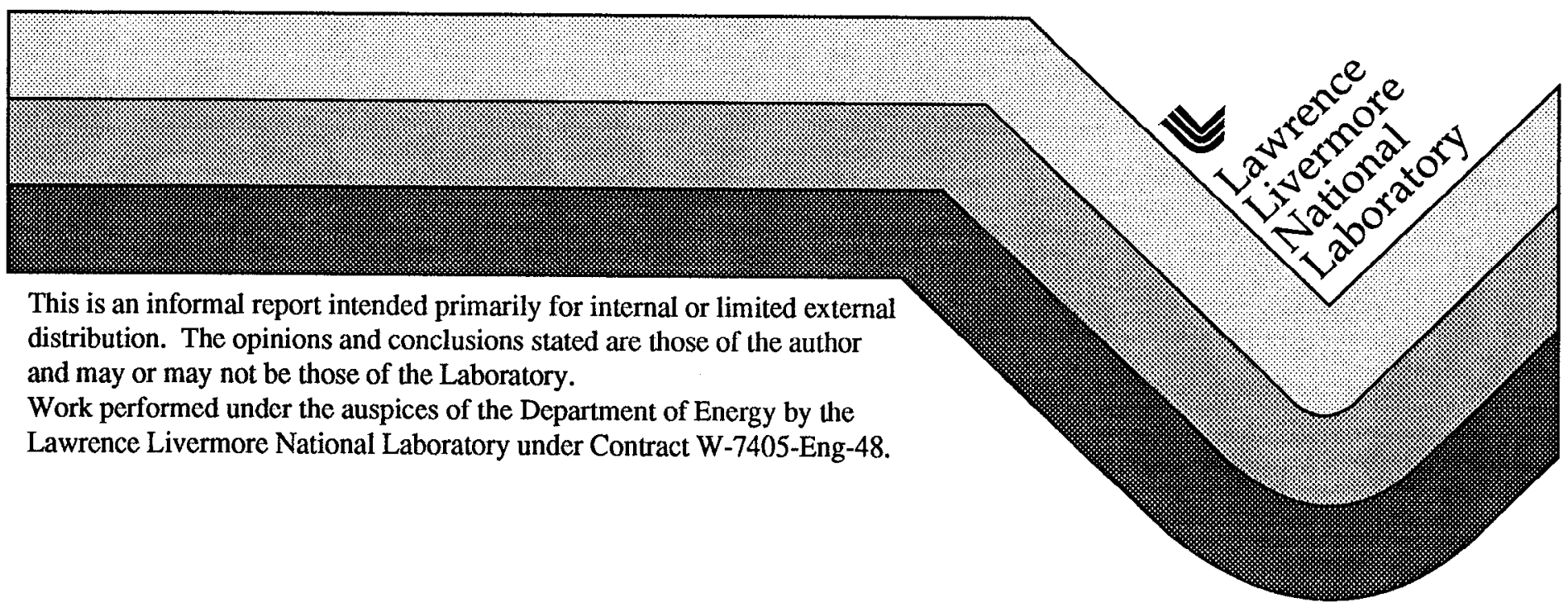




\section{DISCLAIMER}

This document was prepared as an account of work sponsored by an agency of the United States Government. Neither the United States Government nor the University of California nor any of their employees, makes any warranty, express or implied, or assumes any legal liability or responsibility for the accuracy, completeness, or usefulness of any information, apparatus, product, or process disclosed, or represents that its use would not infringe privately owned rights. Reference herein to any specific commercial product, process, or service by trade name, trademark, manufacturer, or otherwise, does not necessarily constitute or imply its endorsement, recommendation, or favoring by the United States Govemment or the University of Califormia. The views and opinions of authors expressed herein do not necessarily state or reflect those of the United States Government or the University of California, and shall not be used for advertising or product endorsement purposes.

This report has been reproduced directly from the best available copy.

Available to DOE and DOE contractors from the Office of Scientific and Technical Information P.O. Box 62, Oak Ridge, TN 37831

Prices available from (615) 576-8401, FTS 626-8401

Available to the public from the

National Technical Information Service

U.S. Department of Commerce

5285 Port Royal Rd.

Springfield, VA 22161 


\title{
High Intensity Physics with a Table-Top 20 TW Laser System
}

\author{
T. Ditmire, and M. D. Perry \\ Laser Science and Technology Program \\ Final Report for LDRD project 98-ERD-084
}

Recent experiments at LLNL as well as at a number of facilities and universities around the world have demonstrated the utility of short pulse $(<100 \mathrm{fs})$ very high peak power $\left(>10^{12}\right.$ W) lasers based on chirped pulse amplification (CPA) in a number of quite interesting applications [1]. The development of CPA roughly 13 years ago has fueled a rapid increase in the focused laser light intensity that is accessible [2]. This development has culminated in the Petawatt laser currently in operation at LLNL. The most important uses of these lasers have only recently come to light, however, and include such applications as the production of very high temperature, high density plasmas and the production of pulsed $\mathrm{x}$-rays with pulse width below $1 \mathrm{ps}$. For example ultra-high intensities focused on solid targets can produce $\mathrm{MeV}$ energy $\mathrm{x}$-rays in sufficient quantities to potentially permit time resolved radiography of hydrodynamic tests. Another application includes the creation of plasmas whose conditions are relevant to astrophysical studies.

The purpose of this project was to develop a high peak power laser system (100 TW) and begin initial high intensity experiments that exploit its short pulse width (30 fs) and high repetition rate $(1-10 \mathrm{~Hz})$. Such a laser system presents unique capabilities such as permitting ultrafast time-resolved plasma physics experiments by probing the plasma with the $30 \mathrm{fs}$ laser pulse. The high repetition rate also allows detailed, systematic studies of phenomena, not possible with large, single shot laser systems.

During the previous year we have made good progress on the development of the laser. We have demonstrated the production of pulses up to the $5 \mathrm{TW}$ level at $10 \mathrm{~Hz}$ and have installed an additional amplifier to take the system to $20 \mathrm{TW}$. We have pulse compressed the pulses to $30 \mathrm{fs}$ and have developed a number of diagnostics to characterize the laser prepulse. During this year we have also activated a target chamber to begin plasma physics experiments in gas jet targets.

The laser itself is currently being constructed to the $0.6 \mathrm{~J}, 30 \mathrm{fs}$ level ( $20 \mathrm{TW}$ of peak power) and operates at a repetition rate of $1 \mathrm{~Hz}$. This system is shown in figure 1. The laser is based on the technique of chirped pulse amplification, the technique now widely used in virtually all short pulse laser systems currently operational including the Petawatt laser. The front end of the laser begins with self-modelocked Ti:sapphire oscillator which produces a beam of pulses with a width near $15 \mathrm{fs}$. The oscillator pulses are stretched in a single grating pulse stretcher to $600 \mathrm{ps}$. The stretched pulses are amplified in regenerative amplifier and two four pass amplifiers. The next stage of amplification (currently under construction) is a three pass amplifier. This is pumped from both sides with a total of $4 \mathrm{~J}$ of green laser light which is produced by a LLNL constructed Nd:YLF laser. The final pulse energy before compression will be near $1 \mathrm{~J}$ and compressed in a vacuum chamber to avoid deleterious break-up of the laser due to nonlinear effects in air, yielding $0.6 \mathrm{~J}$ pulses at $1 \mathrm{~Hz}$. We have demonstrated compression of the laser pulses to $30 \mathrm{fs}$ at the $1 \mathrm{TW}$ level and will demonstrate 20 TW later in FY99. 


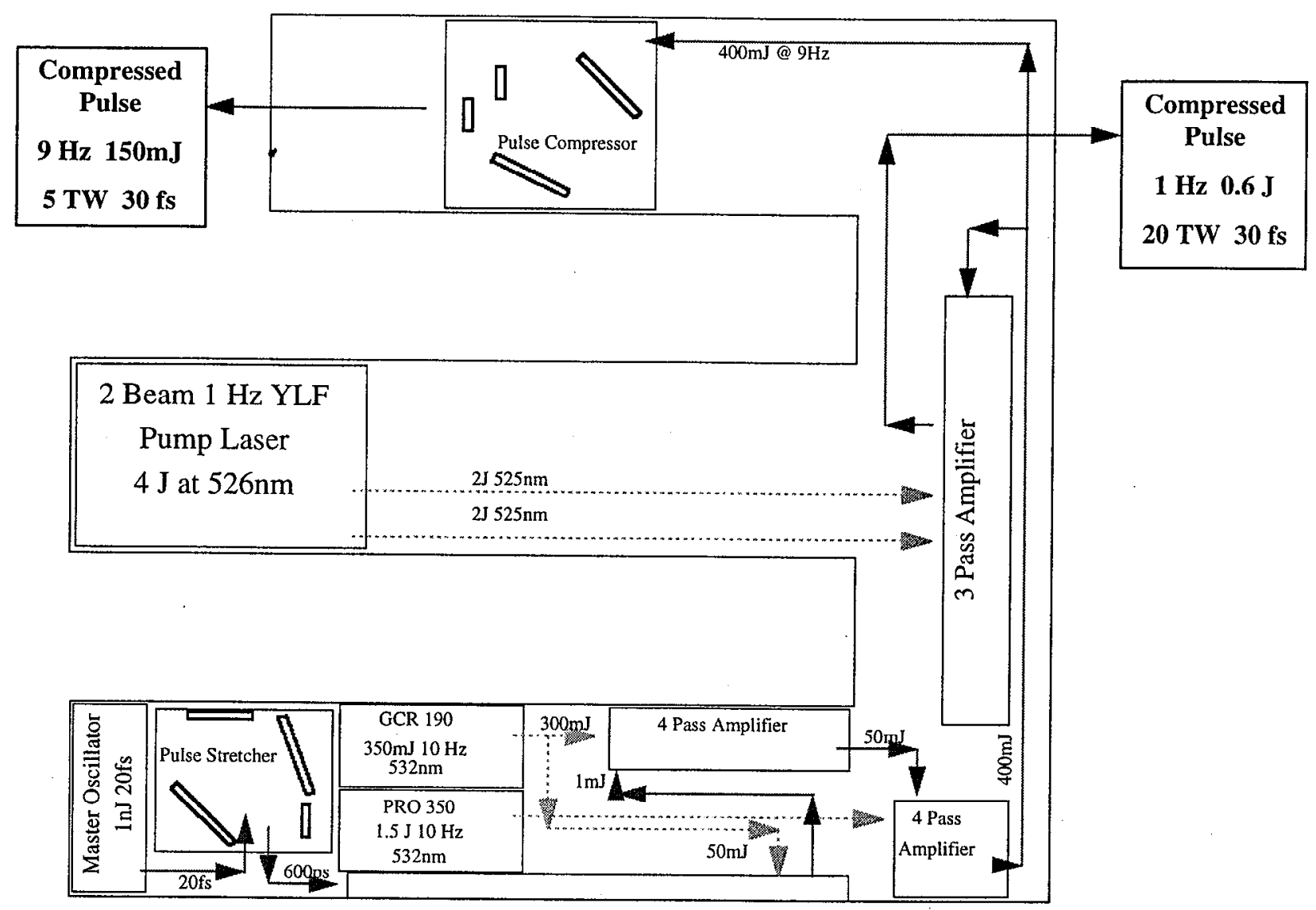

Figure 1: Schematic of the 20 TW Falcon laser currently under construction.

The recompression performance of the laser is illustrated in figure 2 . The selfmodelocked Ti:sapphire oscillator yields $20 \mathrm{fs}$ transform limted pulses. The interferometric autocorrelation of these pulses is shown in the upper left in figure 2. These pulses are stretched in a LLNL designed pulse stretcher [3] which passes $100 \mathrm{~nm}$ of bandwidth. The spectrum of the stretched pulses is also shown in figure 2. After amplification, gain narrowing reduces the pulse bandwidth from $32 \mathrm{~nm}$ (full width at half maximum) to $27 \mathrm{~nm}$. The recompressed pulse autocorrelation at the $150 \mathrm{~mJ}$ level is shown in the bottom right section of the figure. We compress to under $35 \mathrm{fs}$, assuming a Gaussian deconvolution. This pulse width is bandwidth limited (as indicated by the near transform limited value of $\Delta v \Delta \tau=$ $0.43)$.

One of the first set of experiments we have performed has involved the study of radiative blast waves in a low density gas. Understanding such radiative blast waves is crucial to understanding the structure of the interstellar medium [4]. To create a radiative blast wave we have focused the $1 \mathrm{TW}$ laser pulse into a clustering gas (such as argon or xenon) and created a high temperature plasma filament. Because of the low density of the gas and high temperature of the plasma created by the $30 \mathrm{fs}$ laser, radiation transport is sufficiently strong to ionize the gas ahead of the shock and affect the shock propagation. 


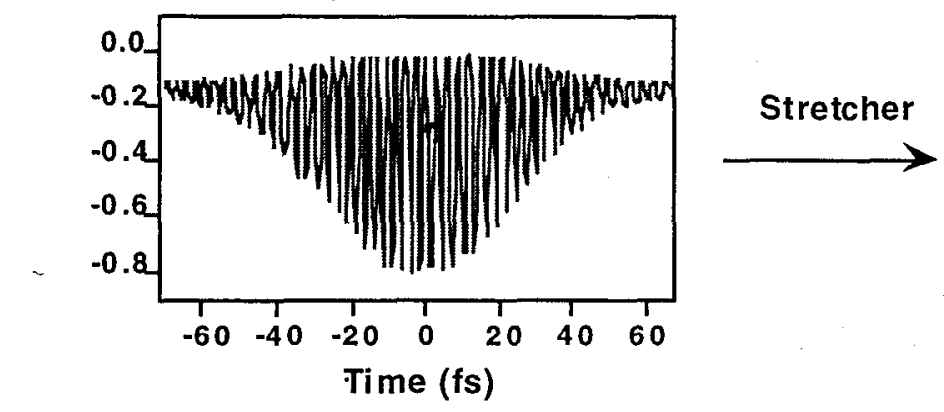

Stretched Laser Pulse Spectrum
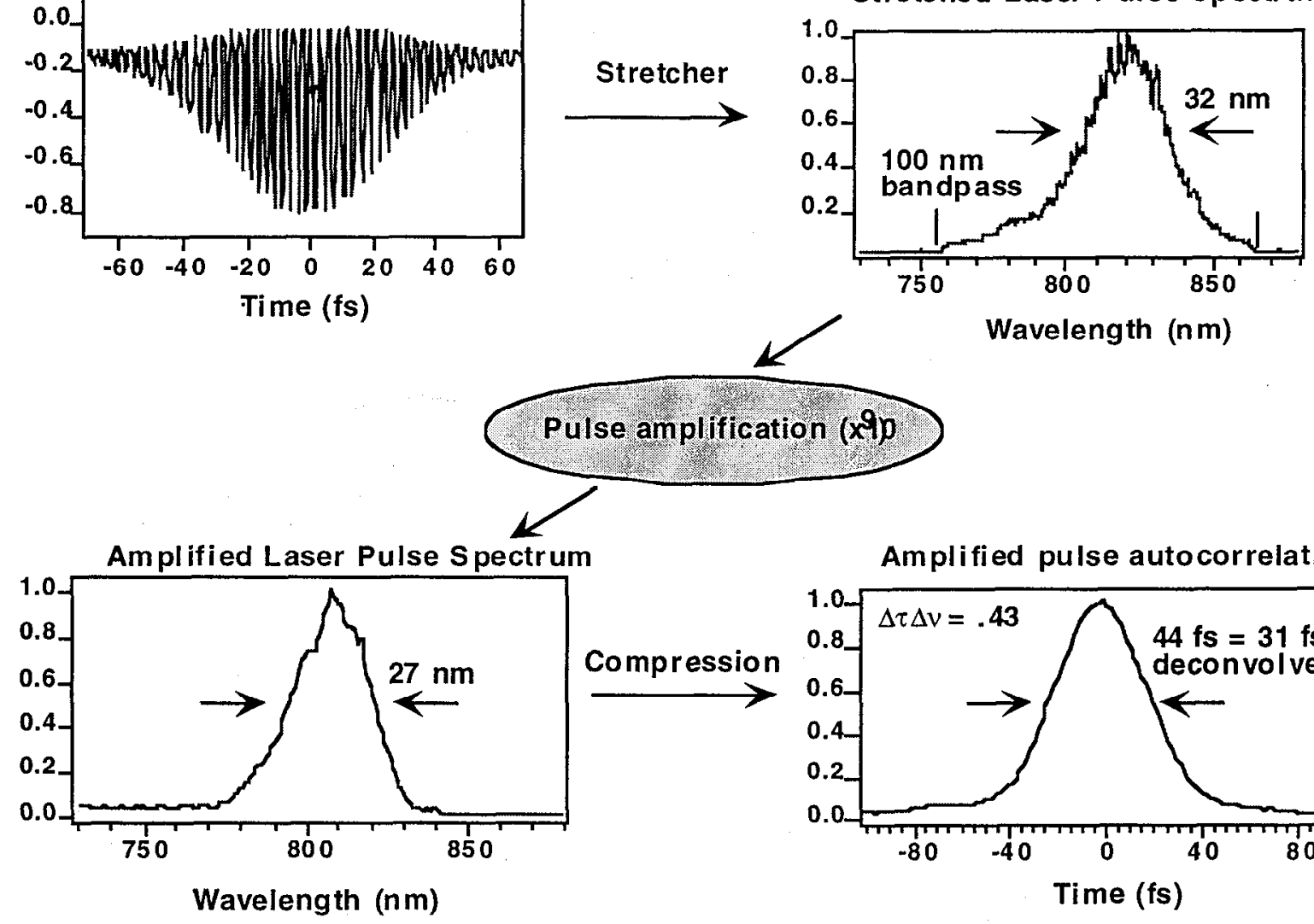

Amplified pulse autocorrelatior

Figure 2: Laser pulse autocorrelations and spectra before and after amplification in the Falcon laser to the $300 \mathrm{~mJ}$ level (150 mJ after compression).

To experimentally characterize the development of strong blast waves in a laser heated clustering gas, we have conducted a series of pump-probe style experiments to characterize the shock profiles [5]. For these studies we utilized laser pulses with energy up to $20 \mathrm{~mJ}$ and pulse width of $30 \mathrm{fs}$. These $820 \mathrm{~nm}$ laser pulses were focused with an f/15 lens into the output of a gas jet nozzle producing medium of clusters in either argon or xenon. The experimental set-up is illustrated in figure 3 .

To probe the time evolution of the shock wave created in the gas jet, a small amount of the main laser pulse (4\%) was split off and sent into a probe beam. This pulse traversed a delay leg and illuminated the plasma filament at a right angle to the propagation direction of the main pulse. The plasma was imaged onto a CCD camera (yielding a spatial resolution of $8 \mu \mathrm{m})$. Between the imaging telescope and the CCD detector the probe light passed through a Michelson interferometer with a roof prism in one leg such that probe light which traversed the plasma filament was interfered with reference light which passed below the plasma. The interferograms yield information on the phase shift resulting from the passage of the light through a chord across the cylindrically symmetric plasma. This phase shift can be Abelinverted to yield the radial electron density profile. By varying the length of the probe delay leg, the time evolution of the plasma profile can be simply mapped. 
Figure 4 shows raw interferograms from an argon plasma and a xenon plasma. The initial plasma filament exhibited a diameter of $80 \mu \mathrm{m}$. The length of the filaments were approximately $2 \mathrm{~mm}$. These images show the cylindrical blast wave $6 \mathrm{~ns}$ after the heating pulse has traversed the gas jet. The backing pressure of the gas jet for the xenon data was 200 psi and the backing pressure for the argon data was 600 psi. Because the average atomic density in a sonic gás jet is roughly linear with backing pressure, these two backing pressures were chosen to yield nearly the same mass density. The total absorbed laser energy fraction in the argon gas jet was $\sim 35 \%$ while the absorbed energy fraction in the xenon gas jet was nearly $65 \%$. This corresponds to a deposited energy per unit length of $30 \mathrm{~mJ} / \mathrm{cm}$ in argon and $55 \mathrm{~mJ} / \mathrm{cm}$ in xenon. (The uncertainty in these numbers is high because the exact dynamics of the laser energy deposition as it propagates through the clustering gas jet are not accurately known.)

The deconvolved profiles of the shock waves at the center of the image are also presented in figure 4. The profile of the argon plasma is that of a classic blast wave with a sharp shock front. The blast wave in xenon, however, exhibits a shock with substantial ionization ahead of the front. The extent of this ionization front decreases with time such that it is virtually unobservable after $12 \mathrm{~ns}$. We attribute this ionization precursor to the presence of substantial radiation transport in the xenon plasma. The strong UV and XUV emission of the hot xenon plasma heats the cold, ambient Xe gas prior to the arrival of the blast wave. These initial data seem to confirm that the plasmas created in the clustering gas jet access a regime in which radiation transport becomes important in the evolution of the shock profile.

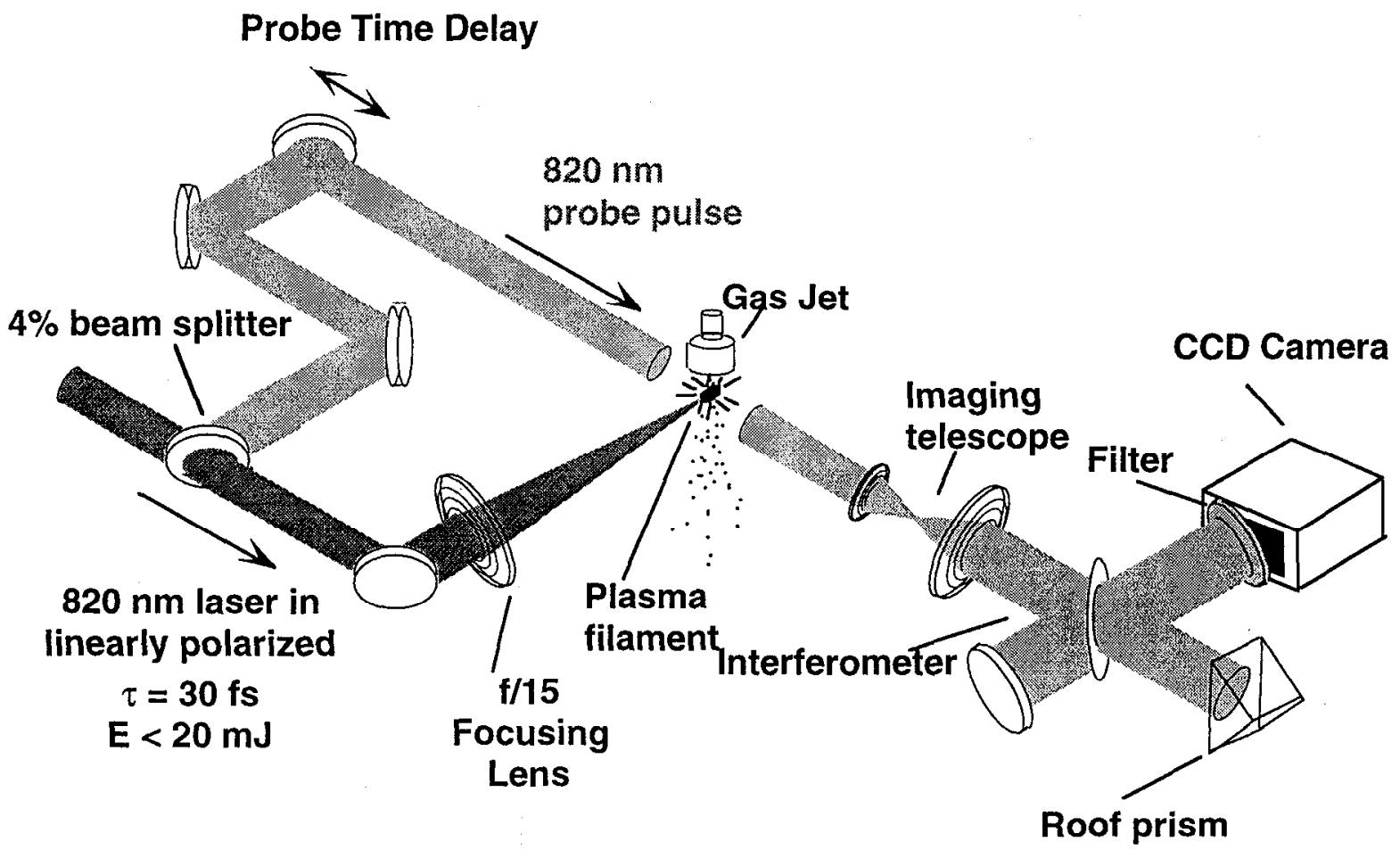

Figure 3: Schematic of the blast wave probing experiment conducted with the Falcon laser. 
In the future, we intend to further pursue these hydrodynamics experiments, including an examination of the effects of an external magnetic field to study radiative magneto-

hydrodynamics. The possibility of conducting fusion experiments with exploding deuterium
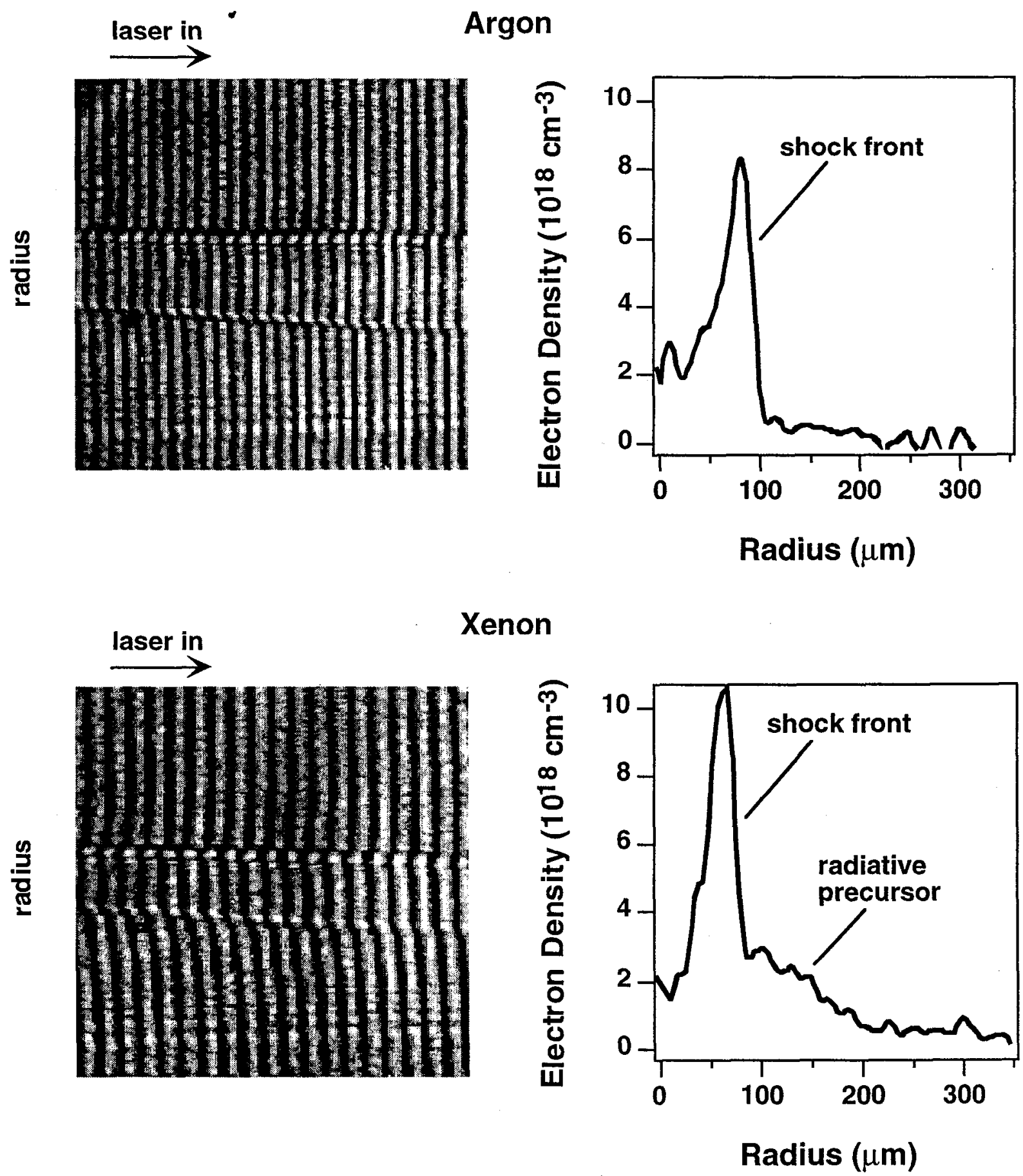

Figure 4: Interferometric images of blast waves produced in argon and xenon gas $6 \mathrm{~ns}$ after being heated by a 30 fs, $17 \mathrm{~mJ}$ laser pulse. The argon gas was produced with 600 psi pressure backing our sonic gas nozzle (with a $0.5 \mathrm{~mm}$ orifice), and the xenon was produced with 200 psi backing pressure. Deconvolved images of the plasma profiles from the center of the images are shown next to the raw data.. 
clusters will be explored as well. We will also study a host of other high intensity experiments, such as $\mathrm{MeV}$ photon production from laser accelerated electrons in solid target interactions, picosecond $\mathrm{x}$-ray production via $\mathrm{K}$-alpha generation in solid targets, and high order harmonic generation in gas jets. Such experiments will hopefully lead the way toward the application of laser-plasma interactions to the development of a laser-based, next generation light source.

\section{References}

1. M. D. Perry and G. Mourou, "Terawatt to Petawatt Subpicosecond Lasers," Science 264, 917 (1994).

2. D. Strickland and G. Mourou, "Compression of Amplified Chirped Optical Pulses," Opt. Comm 56, 219 (1985).

3. P. Banks, V. Yanovsky, M. D. Perry, in preparation for submittal to Opt. Lett.

4. C. F. McKee and B. T. Draine, "Interstellar Shock Waves," Science 252, 397 (1991).

5. T. Ditmire, K. Shigemori, B. A. Remington, K. Estabrook, R. A. Smith, "The Production of Strong Blast Waves Through Intense Laser Irradiation of Atomic Clusters" Ap. J. Supp. to be published. 